\title{
Experimental study the anti-inflammatory and osteo-regenerative qualities of the paste based on symphytum officinale tincture and calcium hydroxide
}

\author{
Iryna Kostyiuk¹, Victor Kostiuk ${ }^{1}$, Halyna Kimak ${ }^{1}$, Yuriy Oktysyuk ${ }^{1}$, Lilia Tarnavska ${ }^{1}$ \\ 1 Ivano-Frankivsk National Medical University, 2 Grushevskogo str., Ivano-Frankivsk, 76000, Ukraine \\ Corresponding author: Iryna Kostyiuk (irinakostyuk@meta.ua)
}

Received 23 April 2021 • Accepted 14 June 2021 • Published 4 August 2021

Citation: Kostyiuk I, Kostiuk V, Kimak H, Oktysyuk Yu, Tarnavska L (2021) Experimental study the anti-inflammatory and osteo-regenerative qualities of the paste based on symphytum officinale tincture and calcium hydroxide. Pharmacia 68(3): 585-590. https://doi.org/10.3897/pharmacia.68.e67774

\begin{abstract}
Replacement of the damaged bone remains actual and is far from being completely solved nowadays, despite its centuries-old history. The purpose of study was to investigate the anti-inflammatory and osteo-regenerative qualities of the paste based on Symphytum officinale tincture and calcium hydroxide in treatment on teeth with chronic granulating apical periodontitis in children and in an experiment on animals. Performed morphological and densitometric studies showed that remodeling of bone injury of the lower jaw in rats occurs almost equally in both experimental and control groups, and a paste based on Symphytum officinale tincture and calcium hydroxide also promotes bone regeneration at the defect site and stimulates osteosynthesis. Analysis of liver tissue, soft and hard tissues of the lower jaw in animals suggest that the proposed paste does not produce any toxic effect and provides significant anti-inflammatory properties. The gained result provide grounds to recommend the developed paste for therapeutic use in children as a temporary root canal sealing material in permanent teeth with granulating apical periodontitis.
\end{abstract}

\section{Keywords}

anti-inflammatory effect, calcium hydroxide, chronic granulating apical periodontitis, experimental study, osteo-regenerative qualities, Symphytum officinale tincture

\section{Introduction}

Despite the fact that treatments for apical periodontitis have long been developed, which traditionally involves the widespread use of antibiotics and synthetic chemotherapeutic agents, some difficulties may arise due to the high variability of microorganisms, which contributes to the constant emergence of new variants resistant to antibacterial agents. In addition, the number of cases reflecting hypersensitivity of the macroorganism to synthetic drugs has increased, which can lead to allergic reactions and other adverse effects (Doña et al. 2014). In this regard, the most promising are methods with the use of herbal pharmacological agents that have different mode of action and suppress root canal infection as well as promote periodontal regeneration (Chomenko et al. 2008).

By the traditional endodontic treatment of immature permanent teeth with apical perodontitis a calcium hydroxide paste is highly recommended. According to data researchers (Kumar et al. 2011; Wikström et al. 2021) calci- 
um hydroxide when placed in canals has a long lasting therapeutic effect therefore providing conditions for the restoration of already damaged tissues of the affected tooth and the subsequent changes in the periodontium, which in the future will ensure its preservation in the dental arch. As the effect of calcium hydroxide is manifested through inflammation, an exacerbation of the pathological process will be an integral part of such treatment (Kim and Kim 2015; Silva et al. 2017). The latter must be remembered when using it for temporary sealing of the root canals in immature permanent teeth (Sheremet and Kyrylenko 2003).

In order to eliminate the negative aspects in management of apical periodontitis in immature permanent teeth and to increase its osteo-regenerative properties, we suggest to add a Symphytum officinale tincture to a calcium hydroxide paste. Symphytum officinale promotes increase of leukocytes at the site of application, thus improving the local immunity, which is important in the healing process. This medicinal plant also expresses good analgetic and hemostatic effect. It is well known that the roots of Symphytum officinale contain large amounts of purine derivatives such as allantoin and allantoic acid that are formed by the breakdown of uric acid in the body of only some animals. Allantoin, on the other hand, is a substance that stimulates cell development and accelerates cell division (proliferation). As a result, the affected tissues recover faster, and regeneration occurs even where the necrosis has reached deep layers. The findings provided by authors (Staiger 2012; Sowa et al. 2018) demonstrated that Symphytum officinale has a multifaceted synergistic effect on the body and this substance is not harmful to humans or animals.

Thereby, it can be assumed that Symphytum officinale tincture as a part of the recommended paste will provide broad anti-inflammatory, necrolytic (mediated antimicrobial), and antihemorrhagic, sedative, immune-stimulating and regenerative effects. Obviously, the symptoms of inflammation caused by calcium hydroxide will decrease and the osteoregenerative properties of the paste, on the other hand, will improve.

Thus, the problem of bone regeneration caused by any injuries is one of the oldest in medicine and, despite its centuries-old history, remains far from being completely solved to this days (Kiyasov and Saleeva 2003). According to authors (Pavlenko et al. 2008; Borysenko and Palamarchuk 2012) it is of a great importance to optimize reparative osteogenesis in general and in dentistry in particular.

The article demonstrates the results of investigations regarding anti-inflammatory and osteo-regenerating properties of the paste based on Symphytum officinale tincture and calcium hydroxide, proposed for the treatment of chronic granulating apical periodontitis in permanent teeth in children.

\section{Materials and methods}

The experimental study was performed on 36 young rats (males) of the Wistar line (weighing 130-150 g), which were on the diet of the vivarium. The animals were divided into experimental and control groups containing 18 rats each. The rats were operated under sterile conditions conducted by ketamine anesthesia. After the skin was managed with alcohol, a scalpel was used to make an incision along the lower edge of the mandible at the region of molars. A longitudinal incision of the buccal muscle was also performed, and then an access to the bone was achieved. A bone defect was simulated by a sharp dental probe, in which the animals of the experimental group were injected with a paste based on Symphytum officinale tincture and calcium hydroxide. Paste with Symphytum officinale Tincture and Calcium Hydroxide contains 55\% alcohol solution (1:10) of Symphytum officinale Tincture and Calcium Hydroxide and is prepared with paste ingredient glycerin.

\section{The composition of the paste}

\section{Symphytum officinale Tincture (1:10) 1.0 \\ Glycerin 0.5 \\ Calcium Hydroxide up to 3.0}

Recipe of Paste based on Symphytum officinale and Calcium Hydroxide

In a container combine $1 \mathrm{ml}$ of Symphytum officinale Tincture (1:10) and $0.5 \mathrm{~g}$ of glycerin and mix ingredients. Gradually add Calcium Hydroxide to form a homogeneous pasty consistency (the amount is approximately $3.0 \mathrm{~g}$ ).

\section{Appearance}

The paste is a homogeneous, plastic, viscous mixture of yellowish color with a slight specific odor. Delamination and mechanical inclusions are not observed, there are no gas evaporation and sludge formation, $\mathrm{pH}=10$.

The control group was operated without the application of paste, the bone defect healed after the formation of a blood clot. The surgical wound was sutured in layers using a polyamide thread and treated with $1 \%$ alcohol solution of diamond green. Rats were fed with raw minced meat to prevent cannibalism triggered by the smell of blood from fresh postoperative wounds immediately after surgery and for the next few days.

Material was collected from animals of both experimental and control groups ( 6 rats each) on $7^{\text {th }}, 14^{\text {th }}$ and $28^{\text {th }}$ days after the surgery. Animals were eliminated from the experiment by decapitation under ketamine anesthesia. The maintenance of rats, their nutrition and experiments with them were carried out in compliance with ethical and legal norms required by the performance of scientific and morphological research, in particular: Addition 4 to the document "Rules for carrying out researches with the use of experimental animals", approved by the order of the Ministry of Health of Ukraine № 755, 12.08.1997. The experimental animals were operated and removed from the experiment in one day, under the same conditions.

After the rats were removed from the experiment, the obtained material was immediately fixed in a $10 \%$ solu- 


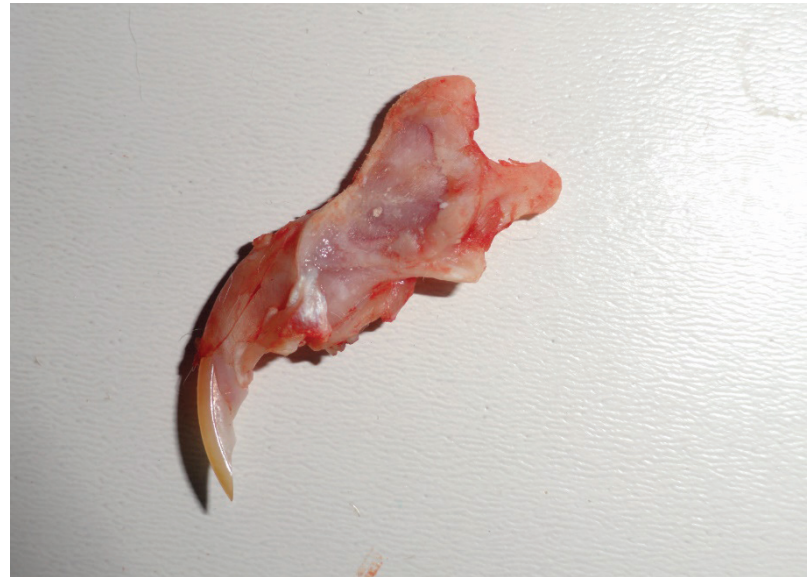

Figure 1. Microphotograph of the mandible with an artificial defect around canines that was filled with the recommended paste in a rat of the experimental group on the $7^{\text {th }}$ day since the beginning of the experiment.

tion of neutral buffered formalin for 48 hours. Further preparation of the material for microscopic investigation was carried out depending on the type of tissue. Thus, to obtain histological sections of the jaw bones (Fig. 1), they were previously decalcified in highly concentrated formic acid diluted with $10 \%$ formalin solution(1:1) for 72 hours.

After decalcification, the jaws were washed in $10 \%$ formalin solution for three days. The prepared bones and adjacent soft and liver tissue were dehydrated in an ascending alcohol battery and embedded in paraffin. Serial histological sections $5 \mu \mathrm{m}$ thick were made on a sled microtome. After the sections were dewaxed, the specimens were stained with hematoxylin and eosin and analyzed on a Carl ZEISS Axiostar plus microscope (Microlmaning $\mathrm{GmbH}$, Germany) equipped with a Canon G 10 camera for digital images.

Mineral density of artificial defects and not affected parts was measured by radiographs of the lower jaws of rats. Radiography was performed on the apparatus «Clinomal PIXEL HF 650» with the following parameters: voltage on the tube $-45 \mathrm{kV}$, current - 25-50 mA, focal length $-100 \mathrm{~cm}$, exposure time was $10-15$ seconds. Radiographs were taken on $24 \times 30 \mathrm{KODAK}$ radiographic medical films. The KODAK-X-RAY automatic, desktop machine was used for radiographs development. Absolute densitometric parameters of the bone mineral density of the lower jaw of rats were measured on the obtained radiographs using an radiographic densitometric device «Densitometer RD501» from KUNTZE (England).

\section{Results and discussion}

Morphological study of soft tissues and bones of the lower jaw and liver in experimental animals was conducted at different time of the experiment to determine osteostimulating qualities of the recommended paste as well as to decide on its general and local toxic effects. Microscopic investigation suggested that the blood vessels of the connective tissues

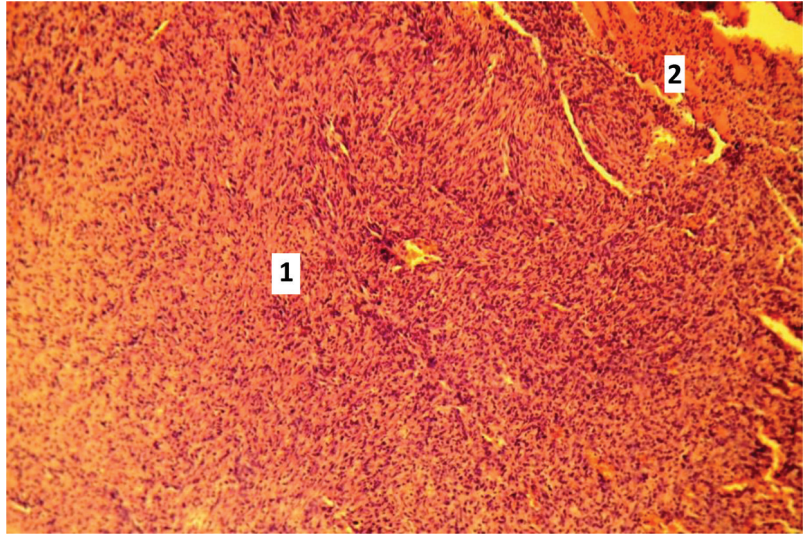

Figure 2. Microphotograph of the adjacent to the site of surgery soft tissues on a fragment of the mandible in an experimental rat 7 days after the begininig of the experiment. The blood vessels of the connective tissue (1) appear full of blood and there is a swelling of the muscle tissue (2). H\&E, Magn.: oc. 10, ob. 10.

appear full of blood and there was a moderate edema of muscle tissue observed on the $7^{\text {th }}$ day after surgery (Fig. 2).

Histological investigation of the adjacent to the area of surgery soft tissues in rats from the control group differed from that in the experimental group by the absence of edema and more pronounced vascular plethora (Fig. 3).

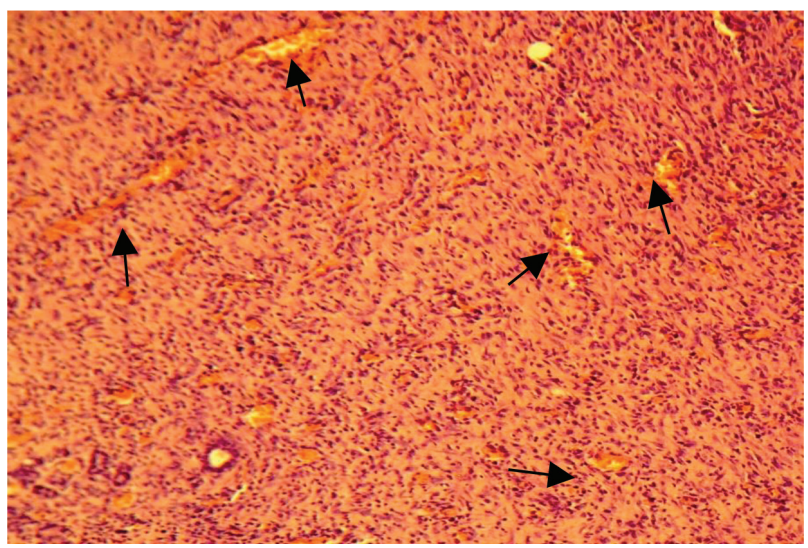

Figure 3. Microphotograph of the adjacent to the site of surgery soft tissues on a fragment of the mandible in a rat from control group 7 days after the begining of the experiment. The blood vessels of the connective tissue (1) appear full of blood ( $\uparrow$ ). H\&E, Magn.: oc.10, ob. 10.

Investigation performed on the $7^{\text {th }}$ day revealed aggregation of polymorphonuclear leukocytes and edema of the tissues at the site of the bone defect with no signs of bone regeneration in both cases when the studied paste was employed or without it (Fig. 4).

However, on the $14^{\text {th }}$ day after surgery the deposition of the newly formed bone islands was visible in rats of the experimental group, in which the paste was employed. Bone remodeling involved pronounced cellular inflammatory reaction as a respond to mechanical damage and chemical irritation to the bone (Fig. 5). At the same terms bone healing in animals of the control group, in which the bone defect repair under a blood clot, comprised a proliferation 


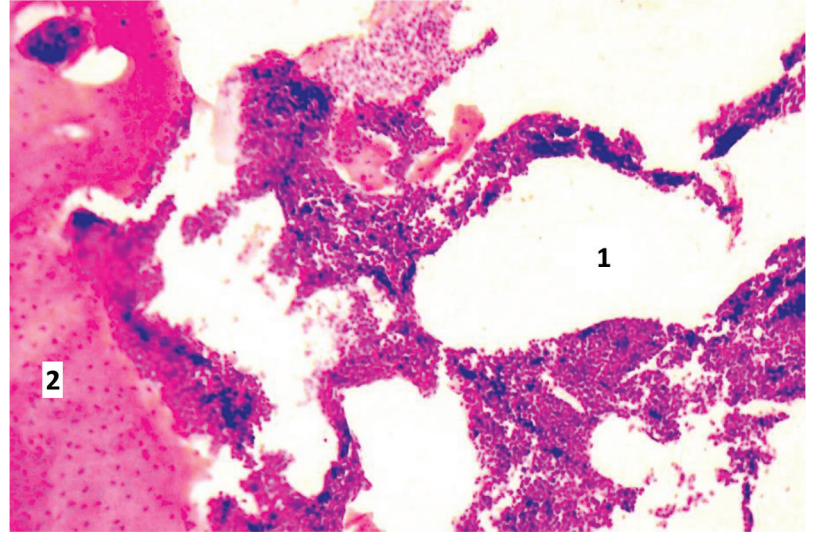

Figure 4. Microphotograph of a fragment of the mandible in a rat from experimental group 7 days after the begining of the experiment. The bone defect is filled with polymorphonuclear leukocytes (1) with edema of the surrounding tissues (2). H\&E, Magn.: oc.10, ob. 40.

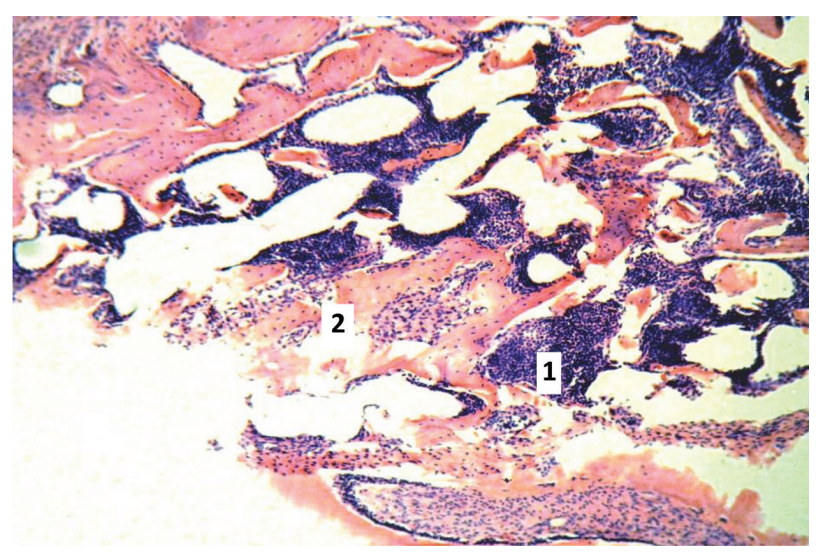

Figure 5. Microphotograph of a fragment of the mandible in a rat from experimental group 14 days after the begining of the experiment. The bone islands are formed (2) among the mixed cellular inflammatory infiltrate (1) and fill the bone defects. H\&E, Magn.: oc.10, ob. 10.

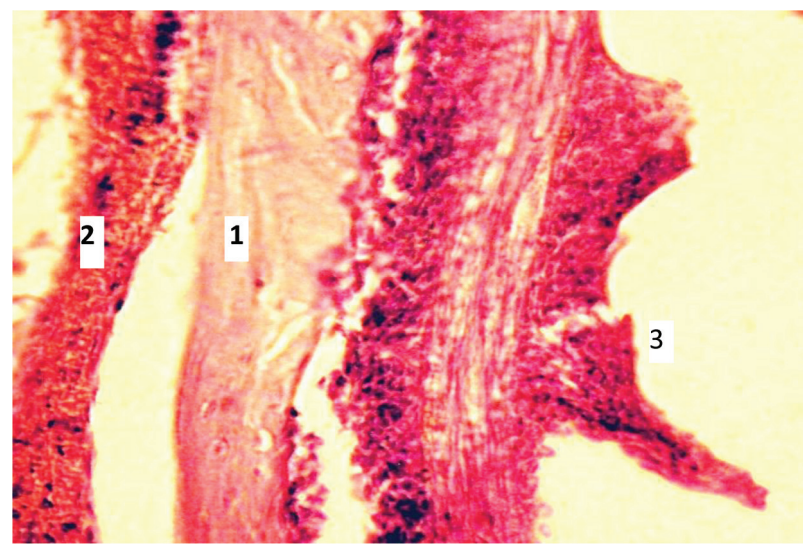

Figure 6. Microphotograph of a fragment of the mandible in a rat from control group 14 days after the begining of the experiment. The connective tissue capsule (1) separates the preserved bone (2) from the bone defect (3). H\&E, Magn.: oc.10, ob. 40.

of fibroblasts and the formation of a connective tissue capsule that separated the damaged area from the preserved bone (Fig. 6).
In this case, as in the experimental group, there was a formation of bone islands among mixed cellular inflammatory infiltrate where the polymorphonuclear leukocytes were joined by macrophages (Fig. 7).

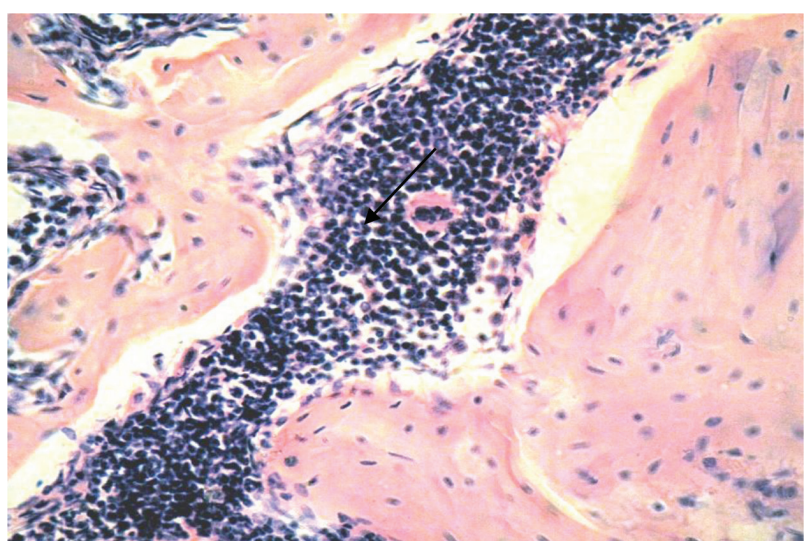

Figure 7. Microphotograph of the defect of the mandible in a rat of the control group on the $14^{\text {th }}$ day from the beginning of the experiment. There is an inflammatory infiltrate consisting of lymphocytes, leukocytes and macrophages found between the islets of newly formed bone tissue ( $\uparrow$ ).H\&E, Magn.: oc.10, ob. 40.

The most pronounced bone regeneration of the mandibles was recorded in animals of both groups on the $28^{\text {th }}$ day after surgery: newly formed bone islands, separated by connective tissue fibers, displaced lymphocytic inflammatory infiltrate from the defect area (Fig. 8).

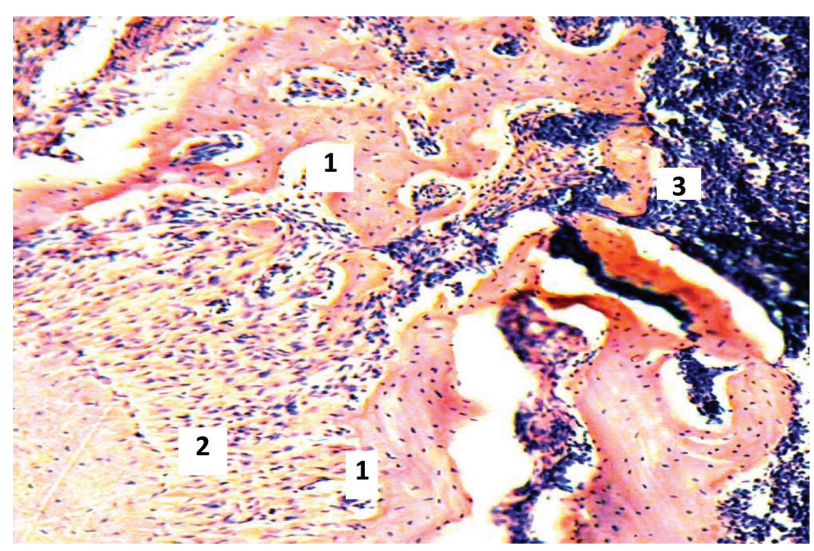

Figure 8. Microphotograph of the defect of the mandible in a rat of the experimental group on the $28^{\text {th }}$ day from the beginning of the experiment. Newly formed bone islands (1), separated by connective tissue fibers and fibroblasts (2), displace lymphocytic inflammatory infiltrate from the defect area (3). H\&E, Magn.: oc.10, ob. 10.

However, bone regeneration in animals of the experimental group was somewhat slower than in the control animals observed 28 days after the beginning of the experiment.The cellular respond to the damage in the control group was less pronounced and there was a fusion of newly developed bone islands that might be explained by the fact that developed paste has high alkaline properties and is perceived as a foreign body (Fig. 9).

It can be summarized that the paste based on Symphytum officinale tincture and calcium hydroxide is not toxic 


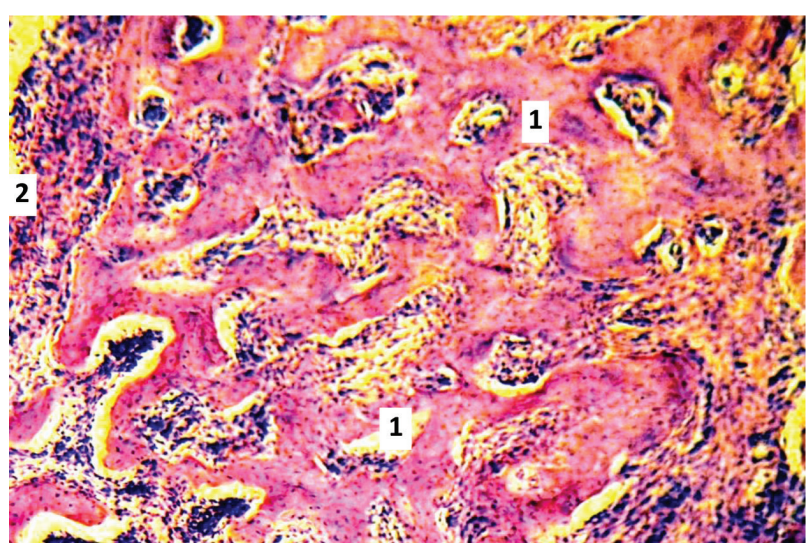

Figure 9. Microphotograph of the defect of the mandible in a rat of the control group on the $28^{\text {th }}$ day from the beginning of the experiment. Newly formed none islands (1) become connected. Inflammatory infiltrate (2) is localized on the periphery of the regeneration zone. H\&E, Magn.: oc.10, ob. 10.

to bones and soft tissues of the jaws, as was there was no evidence of any pathological changes in these tissues in animals of the experimental group. The study suggests that the developed paste promotes bone regeneration at the site of defects and stimulates bone remodeling.

Moreover, to determine the overall toxicity of the recommended paste the examination of liver tissue in experimental animals was performed. The obtained results showed the same morphology of these tissues in both groups of rats, in particular : the liver parenchyma in rats of control and experimental groups is organized of classic hepatic lobules that consist of trabeculae formed by functionally active hepatocytes without pathological changes (Fig. 10).

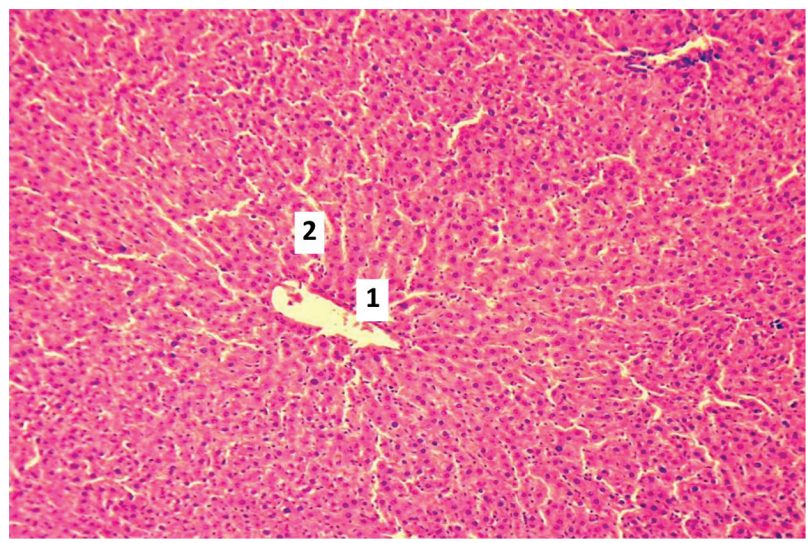

Figure 10. Microphotograph of a fragment of the liver in a rat of the experimental group on the $28^{\text {th }}$ day from the beginning of the experiment. The central vein (1) with radially located trabeculae (2) is defined. H\&E, Magn.: oc.10, ob. 10.

In some cases, histological study demonstrated that the arteries and veins in the hepatic triads (Fig. 11) were full of blood as it might be a result of circulatory disorders due to psycho-emotional instability of the experimental animals.

It can be assumed that the proposed paste does not express a general toxic effect on experimental animals withdrawn from the experiment at different times after surgery.

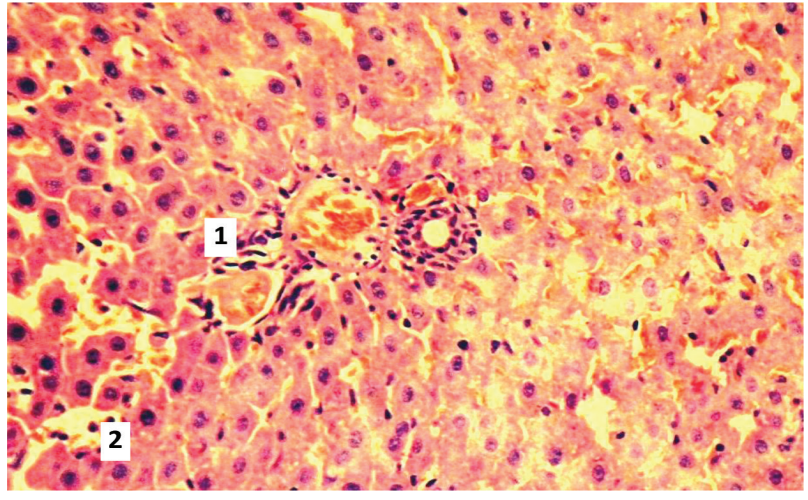

Figure 11. Microphotograph of a fragment of the liver in a rat of the experimental group on the $14^{\text {th }}$ day from the beginning of the experiment. The plethora of arteries (1) and veins (2) in the hepatic triad is determined. H\&E, Magn.: oc.10, ob. 40.

Thus, morphological studies have shown that the developed paste,based on Symphytum officinale tincture and calcium hydroxide and designed for temporary root canal sealing in permanent teeth in children with chronic granulating apical periodontitis, does not produce any local toxic effects (on bone and surrounding soft tissues) and on the body in general (liver tissue).

In addition, the paste has a good anti-inflammatory properties and promotes bone regeneration at the defect site stimulating osteogenesis. These positive biological qualities are encouraging to recommend this paste in pediatric therapeutic dentistry.

Bone densitometry technique was conducted for evaluating bone mass of the affected parts and healthy tissues in experimental animals to assess the healing process of artificial defects filled with the recommended paste and in control group, in which the bone repaired itself by a blood clot. The obtained data are shown in Table 1.

Table 1. Densitometric parameters of mineral density of the lower jaws in rats (absolute units).

\begin{tabular}{lcccc}
\hline $\begin{array}{l}\text { Term after } \\
\text { operation }\end{array}$ & \multicolumn{2}{c}{ Experimental group, $\mathbf{n}=\mathbf{1 8}$} & \multicolumn{2}{c}{ Control group, $\mathbf{n}=\mathbf{1 8}$} \\
\cline { 2 - 5 } & $\begin{array}{c}\text { healthy bone, } \\
\mathbf{n}=\mathbf{6}\end{array}$ & $\begin{array}{c}\text { defect area, } \\
\mathbf{n}=\mathbf{6}\end{array}$ & $\begin{array}{c}\text { healthy bone, } \\
\mathbf{n}=\mathbf{6}\end{array}$ & $\begin{array}{c}\text { defect area, } \\
\mathbf{n}=\mathbf{6}\end{array}$ \\
\hline 7 day & $-0,09 \pm 0,02$ & $-0,38 \pm 0,03^{* * *}$ & $-0,12 \pm 0,01$ & $-0,38 \pm 0,03^{* * *}$ \\
14 day & $-0,21 \pm 0,04$ & $-0,47 \pm 0,03^{* * *}$ & $-0,24 \pm 0,07$ & $-0,46 \pm 0,06^{*}$ \\
28 day & $-0,23 \pm 0,02$ & $-0,41 \pm 0,04^{* *}$ & $-0,10 \pm 0,02$ & $-0,30 \pm 0,03^{* * *}, \mathbf{A}$ \\
\hline
\end{tabular}

Remarks: 1. $\mathrm{p}_{1}<0,05-^{*}, \mathrm{p}<0,01-^{* *}, \mathrm{p}_{1}<0,001-^{* * *}$-probability of data by comparison with a norm.

2. $\mathrm{p}_{2}<0,05-\mathbf{\Delta}$ - probability of data by comparison of information obtained on the $14^{\text {th }}$ day after surgery with those gained on the $7^{\text {th }}$ day.

Analysis demonstates that optical density of the bone tissues in zone of artificial defects was the same on the $7^{\text {th }}$ day of experirement in both groups. On the other hand, a significant difference was revealed when comparing the gained result with the mineral density of healthy bones in both groups $(\mathrm{p}<0,001)$.

The further study indicates that 14 days after surgery the bone density of defects was definitely reduced in the experimental group by comparison with the data on the $7^{\text {th }}$ day and differed from the information obtained on uninvolved bone tissue in 2.24 times $(p<0,001)$. Similar results were ob- 
served also in the control group, but the difference between defects and healthy tissues in this group was less than in the experimental group in 1.92 times $(\mathrm{p}<0,05)$, which suggests that the bone heals faster in animals of the control group.

Bone mineral density appear to have tendency to increase on the $28^{\text {th }}$ day of the study in both experimental and control groups. Indicators of optical density in bones at zones of the defect which healed influenced by paste,were insignificantly higher than those obtained in this group on the $14^{\text {th }}$ day, and the difference remained significant compare to healthy tissue.

However, in those animals in which the defect in the lower jaws healed naturally, these values were in 1.53 times $(\mathrm{p}<0.05)$ higher on the $14^{\text {th }}$ day .

Collation of density with healthy bone tissue was 3.0 times $(p<0.001)$ and was greater and more significant than the same difference in the experimental group (1.78 times; $p<0.01$ ), which proves a positive effect of recommended paste on bone repair.

\section{Conclusion}

1. Morphological studies of liver, soft tissues and bones of the lower jaws in animals revealed the absence of

\section{References}

Borysenko AV, Palamarchuk SI (2012) Administration of paste for temporary root canal sealing in treatment of chronic apical periodontitis. Modern stomatology 2: 9-10.

Chomenko LA, Bidenko NV, Zelenkova AI (2008) Medical interventions in the root canals of deciduous teeth. Part 2: how to treat? Modern dentistry 3: 66-73.

Doña I, Barrionuevo E, Blanca-Lopez N, Torres MJ, Fernandez TD, Mayorga C, Canto G, Blanca M (2014) Trends in hypersensitivity drug reactions: more drugs, more response patterns, more heterogeneity. Journal of Investigational Allergology and Clinical Immunology 24(3): 143-153.

Kim D, Kim E (2015) Antimicrobial effect of calcium hydroxide as an intracanal medicament in root canal treatment: a literature review Part II. in vivo studies. Restorative dentistry and endodontics 40(2): 97-103. https://doi.org/10.5395/rde.2015.40.2.97

Kiyasov AP, Saleeva HT (2003) Bone tissue and dynamics of bone repair in experiment after implantation in case of osteoporosis. Dentistry for everyone 3: 36-39.

Kumar R, Patil S, Hoshing U, Medha A, Mahaparale R (2011) MTA Apical Plug and Clinical Application of Anatomic Post and Core for Coronal Restoration: A Case Report. Iranian endodontic journal 6(2): 90-94. general and local toxicity of the proposed paste and indicated its anti-inflammatory properties as there was a decrease of cell population responsible for local immunity. The exposure of the paste has also increased. The investigation has shown that the paste promoted bone regeneration, which was confirmed by the formation of bone islands at the sites of defects beginning from the $14^{\text {th }}$ day of the experiment.

2. Densitometric parameters of bone mineral density in rats indicate that there was no differences in tissue density at all times after surgery in experimental and control group during the regeneration of artificial defects with administration of the proposed paste and in those that healed naturally.

3. Conducted morphological and densitometric studies have shown that bone remodeling is almost the same in both the experimental and control groups, and paste based on Symphytum officinale tincture and calcium hydroxide promotes bone regeneration at the defect site and stimulates osteosynthesis.

4. The obtained data provide the grounds to recommend this paste intended for root canal sealing in permanent teeth with chronic granulating apical periodontitis in children for wide administration in practical medicine.
Pavlenko AV, Horban SA, Ilyk RR, Shterenberh A (2008) Osteoplastic materials in dentistry: past, present, future. Modern dentistry 4: 103-109. Sheremet VM, Kyrylenko NB (2003) Non-operative treatment of radicular granulomas and cystogranulomas. Dentist 12: 34-36.

Silva LA, Romualdo PC, Silva RA, Souza-Gugelmin MC, Pazelli LC, De Freitas AC, Faria G, Nelson-Filho P (2017) Antibacterial effect of calcium hydroxide with or without chlorhexidine as intracanal dressing in primary teeth with apical periodontitis. Pediatric Dentistry 39: 28-33.

Sowa I, Paduch R, Strzemski M, Zielińska S, Rydzik-Strzemska E, Sawicki J, Kocjan R, Polkowski J, Matkowski A, Latalski M, Wójciak-Kosior M (2018) Proliferative and antioxidant activity of Symphytum officinale root extract. Natural Product Research 32(5): 605-609. https:// doi.org/10.1080/14786419.2017.1326492

Staiger C (2012) Comfrey: a clinical overview. Phytotherapy Research 26(10): 1441-1448. https://doi.org/10.1002/ptr.4612

Wikström A, Brundin M, Lopes MF, El Sayed M, Tsilingaridis G (2021) What is the best long-term treatment modality for immature permanent teeth with pulp necrosis and apical periodontitis? European Archives of Paediatric Dentistry. https://doi.org/10.1007/s40368020-00575-1 